\title{
Context-based decision support to form relevant groups of learners
}

\author{
Elsa Negre \\ Paris-Dauphine University, PSL Research Universities \\ CNRS UMR 7243 LAMSADE, \\ Paris, France \\ elsa.negre@dauphine.fr
}

\author{
Marie-Hélène Abel \\ Sorbonne Universités, Université de Technologie de Compiègne, \\ CNRS UMR 7253 Heudiasyc, \\ Compiègne, France \\ marie-helene.abel@hds.utc.fr
}

\begin{abstract}
Working in groups aims to exceed the result that could be obtained by the simple sum of results achieved individually. To this end, the definition of the group is a key element: How to choose the members of the latter, on what criteria to identify them? In our work we focus on the process of forming groups of learners taking into account the characteristics of learners and more generally the context in which they evolve. We specify what we mean by context before presenting our context-based decision support to form relevant groups of learners. Using a motivating example, we illustrate how to use the multi-criteria classification process for decision support. We then discuss its applicability within a collaboration platform developed from a semantic model.
\end{abstract}

Index Terms-Context-aware computing, Decision support, Multi-Criteria Decision Analysis, Dominance-based Rough Set Approach, Collaborative Work Environment, e-learning

\section{INTRODUCTION}

Working in groups was facilitated with the advent of Web 2.0 technologies whose use for this purpose, becomes more widespread. Their use makes it possible to collect various and varied data (Big Data era) that can be exploited for different purposes such as decision support. If the interest to work in a group is not to prove for the realization of certain tasks, the achievement of intended results and/or the success of the collaboration depends on the composition of the group. The process of developing the latter remains complex. Group composition is usually considered in terms of how individual member characteristics will affect group cohesion or compatibility and subsequently how the group interacts. These charateristics is what we call in this paper: Contextual information/data of the learner, or learner's context. Group composition affects many aspects of the goal achievement, such as how efficiently group members work together and how much relevant knowledge they can share. Thus, when deciding how to compose groups for a project, some tasks are necessary, some are dependent on learner's context such that: (a) Define the project learning objectives (depending on the learners), (b) decide how to configure the group (depending on some constraints (technical, linked to learner's context, ...)), (c) select group members (the characteristics of a group are not the sum of the characteristics of each constituent), (d) develop a contingency plan in case group membership changes during the project; And some are contextual information such that: (e) Identify characteristics of group members relevant to the project learning objectives.

In the framework of our work, we are interested in helping the composition of a learning group for a project. The aim is to propose an approach to group members with recommendations to configure the groups according to some constraints and contextual information; And thus, propose a context-based decision support to form relevant groups of learners. Thus, the framework of our approach consists in the formation of groups of learners using the same learning/collaborative platform where the manager/teacher must constitute groups of learners according to different contextual information and constraints concerning the learners and/or groups he needs to define. We consider that we are able, through the collaborative platform to offer him a set of (contextual) information about learners that he can use to define his choice/selection criteria that will impact the composition of groups.

Finally, our goal is to find a set of learners (grouped into groups) that maximizes the match with these criteria and respects the constraints of the manager/teacher. Thus, our context-based decision support proposes an ordered set of possibilities/alternatives to group the learners (this set can be empty). The manager/teacher can then:

- Validate an alternative of the set by having access for each learner to the contextual information that led to its selection in this group,

- Deny all possibilities/alternatives. In this case, the manager/teacher will be asked to lift/harden criteria and/or constraints to restart the search for groups.

This article is organized as follows: After presenting the state of the art on the notions of groups in a learning/collaborative platform, the activities and traces of interactions between the learners and; The context of the learner, we introduce in section III our context-based decision support to form relevant groups of learners based on contextual information. Then we illustrate it via a motivating example in section IV and a possible applicability in section V before concluding and presenting some research perspectives in section VI. 


\section{RELATED WORKS}

\section{A. e-learning}

e-learning is the set of solutions and means for learning via electronic media. "This allows users to fruitfully gather knowledge and education both by synchronous and asynchronous methodologies to effectively face the need to rapidly acquire up to date know-how within productive environments" [13]. Asynchronous e-learning supports work relations among learners and with teachers, event when participants can not be online at the same time. Thus, the learner learns at his own pace by having full control over his training and the ability to connect to his convenience. In contrast, synchronous e-learning allows the development of interactions between learners (thus limiting isolation) and avoid frustration by asking/answering questions in real time [11]. Asynchronous and synchronous e-learning complement each other [11].

In addition, learners who have different natures must sometimes be grouped to carry out work. Grouping can be globally divided into homogeneous grouping and heterogeneous grouping $^{1}$. The difference between homogeneous and heterogeneous grouping consists in creating a group of learners presenting or not a similar level of characteristics.

In the field of e-learning, research has led to the development of computer-based tools that support automatic group formation: Learners can be matched to peer learners for a specific task upon request [10]; [12] uses questionnaires and thinking style scores of students as a single point along three orthogonal vectors; [16] and [8] are based on homogeneous and/or heterogeneous grouping by selecting multiple criteria. [2] and [5] have examined how group composing characteristics such as gender, ethnic status, social economic status, and personality type affect learning performance and cooperative interaction.

Most of the works mentioned above group learners based on characteristics or criteria for which they have scores coming from questionnaires, assessments, ... In the context of computer-based learning environment, some information/data can be obtained/inferred through the platform. Moreover, by the time learners interact, their activity traces can be used to indicate their activeness and levels of cognition.

\section{B. Activity and traces}

Learners exchange and get new knowledge by realizing activities together. These activities leave traces. Indeed, a digital trace not only contains the values from the environment properties but also the result of a systematic recording of learners' interactions with the environment.

In a Web-based Collaborative Working Environment, interactions facilitate information sharing. Recording the interactions could constitute an interaction trace. [4] defined such a trace as "histories of users" activities collected in real time from their interaction with the software. It could be used to

\footnotetext{
${ }^{1}$ Difference Between Heterogeneous And Homogeneous Grouping, http://resumes-for-teachers.com/blog/interview-questions/difference-betweenheterogeneous-and-homogeneous-grouping/, 2016
}

identify learners' working experience. Thus, once recorded, traces can be considered as a type of resources in the information system. It could specify interactions users have with the software but also interactions users have with other users by means of the software. Thus, for example, from the user's interaction traces with the system (time/place of the connection, participation (active/inactive), ...), it is possible to infer information as to its way of working (synchronous/asynchronous, leader/participant, ...). If interactions are well described from an appropriate semantic model, collected traces may specify the Learner-User's activities: LU consulted the resource R about the subject $\mathrm{S}$ the day $\mathrm{D}, \mathrm{LU}$ put a comment about the resource $R$ for the members of the group $G$ the day $D, \ldots$ In this article, we consider that Learners-Users' traces can be properly collected and represent activities that Learners-Users did with the support of the Web-based Collaborative Working Environment.

\section{Context}

The most widely accepted definition of context is probably the one proposed in [6]: "Context is any information that can be used to characterize the situation of an entity. An entity is a person, place, or object that is considered relevant to the interaction between a user and an application, including the user and applications themselves." Many categorizations have been proposed to describe what concretely is the context of a user. Some of them for specific domains like contextual information retrieval [15] or context-aware recommender systems [3], and some of them more generally for contextual applications [6].

In e-learning, the context of the learner corresponds to any information that can be used to characterize the situation of the learner, including his/her identity, his/her knowledge/skills and his/her interaction traces.

\section{OUR METHOD TO FORM RELEVANT GROUPS OF}

\section{A. Preliminaries} LEARNERS

Recently, [7] proposed a new proposition of context factors categorization $^{2}$ that (1) satisfies the definition of [6], (2) improves the previous propositions, (3) allows to work with context in different levels, and (4) allows its application to a large spectrum of application domains.

This is a hierarchical categorization with three principal categories of context:

1) The physical context represents all aspects that can be influenced by the geographic position of the user. There are four units in this category: Temporal unit, Spatial unit, Environmental unit, and Equipment unit.

2) The personal context represents personal information about the user, and has four units: Demographic unit, Social unit (could be identified by the means of digital traces coming from the use of social network, forums, digital collaborative platforms, ...), Psychophysiological unit, and Cognitive unit (could be identified by the

\footnotetext{
${ }^{2}$ This categorization is proposed for context-aware recommender system area but is sufficiently generic to be used in our case.
} 
means of digital traces coming from the use of agenda, digital platform used as support for activities.)

3) The technical context gathers characteristics of the devices used by the user to access the application: Hardware unit and Data unit (could be identified by the means of traces coming from the digital applications used).

It should be noted that each unit will be instantiated according to the case of application, the relevance and the availability of the associated indicators. Moreover, for all context factors, we could obtain such information by the means of digital devices using sensors and recording user' activities in applications.

\section{B. Taking into account the context of learners}

From the categorization of context, within the framework of learning/collaborative platforms, here are the context factors that, from our point of view, are accessible and relevant:

1) Physical context:

- Temporal unit: Connection time, ...

- Spatial unit: Connection place, ...

- Environmental unit: We consider that the learner is alone behind his computer.

- Equipment unit: Books, learning materials, ...

2) Personal context:

- Demographic unit: Date of birth, sex, ...

- Social unit: Some learners are alone, others already belong to groups on the platform.

- Psychophysiological unit: State of mind, motivation, ... We are aware that this information is difficult to obtain but, for example, it would be possible by semantically analyzing the traces of the learner on the platform (via text analysis or others like what is done in social networks with opinion mining).

- Cognitive unit: From our point of view, an effective way to obtain contextual information/data related to the cognitive unit is to analyze learner interaction traces within the platform. In [17], we proposed a criterion for grouping learners for either an homogeneous group or an heterogeneous group. To reach this goal, we model activities of learners from a web-based collaborative environment and a Bayes Classifier was applied for analyzing traces and then our approach recommends how to group learners. Moreover, via the analysis of the learners' interaction traces, it is possible to calculate what we call his synchronism score. For example, a learner who often works in real time with other learners will have many synchronous traces and thus, a higher synchronism score than a learner who works systematically alone and has little real-time interaction with others. Thus, our synchronism score $s_{\text {sync }}$ is defined as follows:

$$
s_{\text {sync }}=\frac{\begin{array}{c}
\text { (number of synchronous traces } \\
- \text { number of asynchronous traces })
\end{array}}{\text { total number of traces }}
$$

where $s_{\text {sync }} \in[-1,1]$ and the higher the score, the more the learner works synchronously.

3) Technical context:

- Hardware unit: We consider here that the learner's material is sufficient/adapted to use the learning/collaborative platform.

- Data unit: The manipulated data correspond to the format of the learning supports (pdf, audio, text, ...). To these data are added those relating to the interaction traces of the learner (each trace of the learner is recorded and thus "transformed" into data) [17].

As already noticed, the context in which learners evolve influences what happens into the group. In the rest of this article, we are not interested in modeling context or representing contextual information/data. But, we focus on our contextbased decision support to form relevant groups of learners. Many context models can (in their most basic form) be reduced to an attribute-value format. In order to be as generic as possible in our proposal, we have chosen this format for the learner's context.

\section{Decision support process}

Decision support is an activity based on models and represents, explicitly or formally, the reality. Multi-criteria decision support is about methods and calculations allowing to choose the optimal solution among a whole set of solutions. The decision in the presence of multiple criteria is difficult because the criteria are often conflicting. For this, several multi-criteria decision support methodologies have been developed [14]. In these methodologies, the preferences of the decision-maker are first elicited on each point of view corresponding to a criterion expressed on a cardinal or ordinal scale. These criteria are then aggregated using multi-criteria aggregation methods. For this, these methodologies also offer several tools to elicit the global preferences of decision-makers to parametrize these methods.

[9] have proposed the Dominance-based Rough Set Approach (DRSA), that is mainly based on substitution of the indiscernibility relation by a dominance relation. In DRSA, data are often presented using a particular form of decision table. Formally, a DRSA decision table is a 4-tuple $S=\langle U, Q, V, f\rangle$, where $U$ is a finite set of objects, $Q$ is a finite set of criteria, $V=\bigcup_{q \in Q} V_{q}$ where $V_{q}$ is the domain of the criterion $q$ and $f: U \times Q \rightarrow V$ is an information function such that $f(x, q) \in V_{q}$ for every $(x, q) \in U \times Q$. The set $Q$ is divided into condition criteria ( $s e t C \neq \emptyset$ ) and the decision criterion (class) $d$. Notice, that $f(x, q)$ is an evaluation of object $x$ on criterion $q \in C$, while $f(x, d)$ is the class assignment (decision value) of the object.

Assuming that the domain of a criterion $q \in Q$ is completely pre-ordered by an outranking relation $\succeq_{q} ; x \succeq_{q} y$ means that $x$ is at least as good as (outranks) $y$ with respect to the criterion $q$. Without loss of generality, it is assumed that the domain of $q$ is a subset of reals, $V_{q} \subseteq \mathbb{R}$, and that the outranking relation is a simple order between real numbers $\geq$ such that the following relation holds: $x \succeq_{q} y \Longleftrightarrow f(x, q) \geq f(y, q)$. 
Let $T=\{1, \ldots, n\}$, the domain of decision criterion, $V_{d}$ consists of $n$ elements and induces a partition of $U$ into $n$ classes $\mathbf{C l}=\left\{C l_{t}, t \in T\right\}$, where $C l_{t}=\{x \in U: f(x, d)=$ $t\}$. Each object $x \in U$ is assigned to one and only one class $C l_{t}, t \in T$. The classes are preference-ordered according to an increasing order of class indices, i.e. for all $r, s \in T$ such that $r \geq s$, the objects from $C l_{r}$ are strictly preferred to the objects from $C l_{s}$.

On the basis of the approximations obtained by means of the dominance relations, it is possible to induce a generalized description of the preferential information contained in the decision table, in terms of decision rules. The decision rules are expressions of the form if [condition] then [consequent], that represent a form of dependency between condition criteria and decision criteria. Procedures for generating decision rules from a decision table use an inductive learning principle. We can distinguish three types of rules: Certain, possible and approximate. Certain rules are generated from lower approximations of unions of classes; Possible rules are generated from upper approximations of unions of classes and approximate rules are generated from boundary regions.

In our case, we propose to use the simplest version of the DRSA approach since in the case of learning/collaborative platforms and in particular for helping to form groups of learners, we have a multi-criteria classification problem. Indeed, we want to assign each learner to a given/predefined group (according to all possible crossings of learners to form groups) by comparing the learners. Thus we begin by defining the possible groups (by crossing all learners) which correspond to the constraints of the manager/teacher then all possible arrangements of these groups.

From this preprocessing, we get the decision table: $S=$ $\langle U, Q, V, f\rangle$ where $U$ is the set of all possible arrangements/alternatives, $Q$ is a finite set of criteria (learner's context), $V=\bigcup_{q \in Q} V_{q}$ where $V_{q}$ is the domain of the criterion $q$ according to the context units and $d$ corresponds to the 4 classes: $\mathrm{Cl}_{4}$ : YES (green), $\mathrm{Cl}_{3}$ : RATHER YES (light green), $\mathrm{Cl}_{2}$ : RATHER NO (light red) and $\mathrm{Cl}_{1}$ : NO (red). Here are the details of these 4 classes:

- $\mathrm{Cl}_{4}$ (YES - green): The alternative (group arrangement) satisfies the manager/teacher's constraints according to the learners' context,

- $\mathrm{Cl}_{3}$ (RATHER YES - light green): The alternative (group arrangement) does not fully satisfy the manager/teacher's constraints according to the learners' context. The solution is however relatively relevant/satisfactory,

- $\mathrm{Cl}_{2}$ (RATHER NO - light red): The alternative (group arrangement) only partially satisfies the manager/teacher's constraints according to the learners' context. This alternative should be avoided.

- $C l_{1}$ (NO - red): The alternative (arrangement of groups) does not satisfy the constraints of the manager/teacher according to the context of the learners. This alternative is the worst.

Subsequently, the decision rules must be defined with the manager/teacher. In order to have a simple approach, accessi-

\begin{tabular}{|c|c|c|}
\hline Name & Synchronism score & City \\
\hline$S t_{1}$ & 0.5 & Paris \\
$S t_{2}$ & -1 & New York \\
$S t_{3}$ & 0 & Paris \\
$S t_{4}$ & 1 & New York \\
$S t_{5}$ & 0 & New York \\
$S t_{6}$ & -1 & Paris \\
\hline
\end{tabular}

TABLE I

CONTEXTUAL DATA OF THE SIX LEARNERS

ble to all, fast and easy to use, we limit ourselves to certain rules (with equally weighted criteria). Certain rules have the following form:

if $f\left(x, q_{1}\right) \geq r_{1}$ and $f\left(x, q_{2}\right) \geq r_{2}$ and $\ldots f\left(x, q_{p}\right) \geq r_{p}$ then $x \in C l_{t}$

In our case, some relatively classic rules are:

- if Criterion $_{1} \geq N O$ or Criterion $_{2} \geq N O$ then Alternative $\in C l_{1}$

- if Criterion $_{1}=R A T H E R N O$ and Criterion $_{2} \leq$ RATHER NO then Alternative $\in \mathrm{Cl}_{2}$

- if Criterion $_{1} \leq R A T H E R N O$ and Criterion $_{2}=$ RATHER NO then Alternative $\in \mathrm{Cl}_{2}$

- if Criterion $_{1}=$ RATHER YES and Criterion $_{2} \leq$ RATHER YES then Alternative $\in \mathrm{Cl}_{3}$

- if Criterion $_{1} \leq R A T H E R Y E S$ and Criterion $_{2}=$ RATHER YES then Alternative $\in \mathrm{Cl}_{3}$

- if Criterion $_{1} \leq Y E S$ and Criterion $2 \leq Y E S$ then Alternative $\in \mathrm{Cl}_{4}$

Of course, these rules can be modified according to the needs of the manager/teacher (weighting of the criteria, ...).

\section{Motivating EXAMPLE}

To illustrate the application of DRSA to multicriteria classification for e-learning/collaborative platform, we propose the following example. Consider that a manager/teacher, $M_{1}$, should split 6 learners into groups. $\left\{S t_{1}, S t_{2}, S t_{3}, S t_{4}, S t_{5}, S t_{6}\right\}$ constitute all learners. A lot of contextual information/data relating to the learners are available like their interaction traces, their age, their geographical location (e.g. real-time GPS data or address given explicitly by the learner on the platform during registration), their sex, ... From this contextual information/data, $M_{1}$ defines two criteria to elaborate the groups, to know:

- Criterion ${ }_{1}$ : The geographical proximity (e.g. kilometer distance between locations) of learners ( $M_{1}$ considers that close geographically learners will have common benchmarks and will also meet to work together).

- Criterion ${ }_{2}$ : The synchronism score of learners $\left(M_{1}\right.$ wants a cumulative synchronism score higher than or equal to 0 ).

In addition, a strong constraint of $M_{1}$ is Constraint $_{1}$ : Each group must be composed of exactly two learners. The table I summarizes the contextual data for the six learners that will be used to check the suitability of the criteria Criterion $_{1}$ and Criterion $_{2}$.

In the preprocessing phase, therefore, we must divide the six learners into groups of two, and then ar- 


\begin{tabular}{|l|c|c|}
\hline Group & $\begin{array}{c}\text { Criterion } \\
\text { (geographic proximity) }\end{array}$ & $\begin{array}{c}\text { Criterion } \\
\text { (cumulative scores } \geq 0)\end{array}$ \\
\hline$G_{1}$ & KO & KO \\
$G_{2}$ & OK & OK \\
$G_{3}$ & KO & OK \\
$G_{4}$ & KO & OK \\
$G_{5}$ & OK & KO \\
$G_{6}$ & KO & KO \\
$G_{7}$ & OK & OK \\
$G_{8}$ & OK & KO \\
$G_{9}$ & KO & OK \\
$G_{10}$ & KO & OK \\
$G_{11}$ & KO & OK \\
$G_{12}$ & OK & KO \\
$G_{13}$ & OK & OK \\
$G_{14}$ & KO & OK \\
$G_{15}$ & KO & KO \\
\hline
\end{tabular}

TABLE II

RESULTS ASSIGNED TO EACH GROUP FOR EACH CRITERION (OF THE MOTIVATING EXAMPLE)

range them for arrangements of three groups. Mathematically, the number of combinations of two elements among six is: $C_{6}^{2}$, i.e. 15 possible combinations of groups of two. So the 15 groups of two possible are:

- $G_{1}:\left(S t_{1}, S t_{2}\right)$

- $G_{2}:\left(S t_{1}, S t_{3}\right)$

- $G_{9}:\left(S t_{2}, S t_{6}\right)$

- $G_{3}:\left(S t_{1}, S t_{4}\right)$

- $G_{4}:\left(S t_{1}, S t_{5}\right)$

- $G_{5}:\left(S t_{1}, S t_{6}\right)$

- $G_{6}:\left(S t_{2}, S t_{3}\right)$

- $G_{7}:\left(S t_{2}, S t_{4}\right)$

- $G_{8}:\left(S t_{2}, S t_{5}\right)$

- $G_{10}:\left(S t_{3}, S t_{4}\right)$

- $G_{11}:\left(S t_{3}, S t_{5}\right)$

- $G_{12}:\left(S t_{3}, S t_{6}\right)$

- $G_{13}:\left(S t_{4}, S t_{5}\right)$

- $G_{14}:\left(S t_{4}, S t_{6}\right)$

- $G_{15}:\left(S t_{5}, S t_{6}\right)$

Then, these 15 groups must be organized in 3tuples (three-group arrangements). In view of the composition of the groups, there are 15 possible arrangements of these groups in 3-tuples such as:

- $A_{1}:\left(G_{1}, G_{10}, G_{15}\right)$

- $A_{2}:\left(G_{1}, G_{11}, G_{14}\right)$

- $A_{3}:\left(G_{1}, G_{12}, G_{13}\right)$

- $A_{4}:\left(G_{2}, G_{7}, G_{15}\right)$

- $A_{5}:\left(G_{2}, G_{8}, G_{14}\right)$

- $A_{6}:\left(G_{2}, G_{9}, G_{13}\right)$

- $A_{7}:\left(G_{3}, G_{6}, G_{15}\right)$

- $A_{8}:\left(G_{3}, G_{8}, G_{12}\right)$

- $A_{9}:\left(G_{3}, G_{9}, G_{11}\right)$

- $A_{10}:\left(G_{4}, G_{6}, G_{14}\right)$

- $A_{11}:\left(G_{4}, G_{7}, G_{12}\right)$

- $A_{12}:\left(G_{4}, G_{9}, G_{10}\right)$

- $A_{13}:\left(G_{5}, G_{6}, G_{13}\right)$

- $A_{14}:\left(G_{5}, G_{7}, G_{11}\right)$

- $A_{15}:\left(G_{5}, G_{8}, G_{10}\right)$

In order to establish the arrangement decision table, it is necessary to assign a result for each criterion and each arrangement. Knowing that an arrangement is a 3-tuple of groups, we must first specify this for each group. Table II shows the result assigned to each group for each criterion. If the criterion is satisfied, the result is OK, otherwise it is KO.

Thus, it is possible to obtain the results for the arrangements according to the following four rules:

- If all are OK then YES

- If all are KO then NO

- If the number of $\mathrm{KO} \geq$ the number of OK then RATHER NO

- If the number of OK $>$ the number of KO then RATHER YES

\begin{tabular}{|c|c|c|c|}
\hline & Criterion $_{1}$ & Criterion $_{2}$ & Decision (d) \\
\hline$A_{1}$ & NO & RATHER NO & NO \\
$A_{2}$ & NO & RATHER YES & NO \\
$A_{3}$ & RATHER YES & RATHER NO & RATHER NO \\
$A_{4}$ & RATHER YES & RATHER YES & RATHER YES \\
$A_{5}$ & RATHER YES & RATHER YES & RATHER YES \\
$A_{6}$ & RATHER YES & YES & RATHER YES \\
$A_{7}$ & NO & RATHER NO & NO \\
$A_{8}$ & RATHER YES & RATHER NO & RATHER NO \\
$A_{9}$ & NO & YES & NO \\
$A_{10}$ & NO & RATHER YES & NO \\
$A_{11}$ & RATHER YES & RATHER YES & RATHER YES \\
$A_{12}$ & NO & YES & NO \\
$A_{13}$ & RATHER YES & RATHER NO & RATHER NO \\
$A_{14}$ & RATHER YES & RATHER YES & RATHER YES \\
$A_{15}$ & RATHER YES & RATHER NO & RATHER NO \\
\hline
\end{tabular}

TABLE III

DECISION TABLE OF THE MOTIVATING EXAMPLE

\begin{tabular}{|c|c|c|c|}
\hline & Criterion $_{1}$ & Criterion $_{2}$ & Decision (d) \\
\hline$A_{1}$ & 0 & 0.25 & 0.125 \\
$A_{2}$ & 0 & 0.75 & 0.375 \\
$A_{3}$ & 0.75 & 0.25 & 0.5 \\
$A_{4}$ & 0.75 & 0.75 & 0.75 \\
$A_{5}$ & 0.75 & 0.75 & 0.75 \\
$A_{6}$ & 0.75 & 1 & 0.875 \\
$A_{7}$ & 0 & 0.25 & 0.125 \\
$A_{8}$ & 0.75 & 0.25 & 0.5 \\
$A_{9}$ & 0 & 1 & 0.5 \\
$A_{10}$ & 0 & 0.75 & 0.375 \\
$A_{11}$ & 0.75 & 0.75 & 0.75 \\
$A_{12}$ & 0 & 1 & 0.5 \\
$A_{13}$ & 0.75 & 0.25 & 0.5 \\
$A_{14}$ & 0.75 & 0.75 & 0.75 \\
$A_{15}$ & 0.75 & 0.25 & 0.5 \\
\hline
\end{tabular}

TABLE IV

DECISION NOTES OF THE MOTIVATING EXAMPLE

Finally, this preprocessing associated with the rules defined in the previous section (Section III.C.) makes it possible to obtain the decision table III.

The arrangements that seem most relevant for $M_{1}$ are $\left\{A_{4}, A_{5}, A_{6}, A_{11}, A_{14}\right\}$. In order to help at best $M_{1}$ a ranking of these proposals would be welcome.

Suppose for each criterion, a note of " 0 " is assigned to the class NO, " 0.25 " to RATHER NO, " 0.75 " to RATHER YES and " 1 " to YES and that the final score assigned to the decision $d$ is the average of the criteria scores. Thus, in our example, the notes of $d$ will belong to the interval $[0,1]$. Table IV summarizes these notes and makes it possible to order the five relevant arrangements.

Finally, $A_{6}=\left\langle G_{2}, G_{9}, G_{13}\right\rangle=$ $\left\langle\left(S t_{1}, S t_{3}\right),\left(S t_{2}, S t_{6}\right),\left(S t_{4}, S t_{5}\right)\right\rangle$ is the best decision for $M_{1}$ according to its two criteria (geographical proximity and synchronism score) and its initial constraint (groups of two learners).

\section{ApPlicABility}

In the best of worlds, the system should be as we have presented it. But in reality, we did not have such a system at our disposal. We are working on the applicability of our 
proposal as part of the MEMORAe project ${ }^{3}$ which sets a collaboration model and a web-based collaborative platform (developed from this collaboration model). The model distinguishes the subject of the collaboration from the collaboration process. Concerning the object of collaboration, it concerns the organisation's own knowledge: What users share or exchange about; And is represented by a shared reference frame. The model of the collaboration process focuses on modelling heterogeneous resource sharing and indexing by the shared reference frame between individuals and groups of individuals within an organization [1]. In the case of a course, the shared reference frame consists in defining the content of the course with the concepts to be learned/understood. The teacher provides learners with heterogeneous teaching resources (e.g. notes, exercises, slideshows, videos, ...) adapted to the public (level and objective of the course). The shared reference frame will also allow learners to add their own resources deemed relevant (shared with group members or members of other groups). Morevoer, the MEMORAe platform allows to consider/record activity traces.

By the means of the semantic model of MEMORAe, it is possible to register all activities made by users such as who adds which kind of resource on which subject with whom and when. It is thus possible to use this activity traces as criterion. Considering our motivating example, $C_{1}$ could be "has already work on the subject X". We could query the activity traces about create, modify, share, add activities about a resource indexed by the concept $X$. Moreover, contextual data/information (as name/id, location, ...) composing the learner's context can be obtained through the MEMORAe platform.

\section{CONCLUSiOn AND FUTURE WORK}

The question of working in a group is no longer an issue today, it is obvious. The question shifted to the formation of a group. The process of forming a group depends on a set of criteria to be taken into account in order to give the group the best chance of achieving the objectives for which it was formed. We can distinguish the criteria on the group itself (number of members, mode of operation, ...) and the criteria on the members that constitute it (age, level of training, ...).

In our work we focus on the process of forming groups of learners taking into account the characteristics of the learners and more generally the context in which they evolve. In this paper we presented a context-based decision support to form relevant groups of learners, i.e., a multi-criteria decision support process for group formation. This process takes into account the criteria and objectives selected by the manager/teacher and makes it possible to establish a set of proposals for this manager/teacher who will then make his decision. One of the strong points of our proposal is the possibility to define criteria based on the activities carried out during the use of a digital platform and recorded in the form of interaction traces. These traces can range from simple logs to much richer information (platform developed from semantic models).

In our future work we intend to apply our context-based decision-making process of forming groups of learners by exploiting semantic interaction traces. We plan to test it as part of the MEMORAe platform [1] and to define criteria such as members who have already worked together on a given topic, intra-group affinity and/or synergy of a group... Finally, more upstream work will also be conducted on the interest, for the group formation, of selected criteria and imposed constraints.

\section{REFERENCES}

[1] Marie-Hélène Abel. Knowledge map-based web platform to facilitate organizational learning return of experiences. Computers in Human Behavior, 51:960 - 966, 2015.

[2] P C Abrami. Classroom connections: Understanding and using cooperative learning. Harcourt College Pub., 1995.

[3] Gediminas Adomavicius and Alexander Tuzhilin. Context-Aware Recommender Systems, pages 217-253. Springer US, Boston, MA, 2011.

[4] Damien Clauzel, Karim Sehaba, and Yannick Priè. Modelling and visualising traces for reflexivity in synchronous collaborative systems. In Proceedings of INCOS: Intelligent Networking and Collaborative Systems, pages 16-23, 2009.

[5] E.G. Cohen. Restructuring the classroom: Conditions for productive small groups. review of educational research. Review of Educational Research, 64(1):1-35, 1994.

[6] Anind K. Dey. Understanding and Using Context. Personal and Ubiquitous Computing, 5(1):4-7, 2001.

[7] Zahra Vahidi Ferdousi, Elsa Negre, and Dario Colazzo. Context factors in context-aware recommender systems. In Atelier interdisciplinaire sur les systèmes de recommandation, 2017.

[8] A. Gogoulou, E. Gouli, G. Boas, E. Liakou, and M. Grigoriadou. Forming homogeneous, heterogeneous and mixed groups of learners. In Proceedings ICUM, pages 33-40, 2007.

[9] Salvatore Greco, Benedetto Matarazzo, and Roman Slowinski. The Use of Rough Sets and Fuzzy Sets in MCDM, pages 397-455. Springer US, Boston, MA, 1999.

[10] J. Greer, G. McCalla, J. Cooke, J. Collins, V. Kumar, A. Bishop, and J. Vassileva. The intelligent helpdesk: Supporting peer-help in a university course. In Proceedings nternational Conference on Intelligent Tutoring Systems, pages 494-503, 1998.

[11] Stefan Hrastinski. Asynchronous and synchronous e-learning : A study of asynchronous and synchronous e-learning methods discovered that each supports different purposes. EDUCAUSE Quarterly, 31(4):51-55, 2008.

[12] S. Lin and C. Sun. Team-forming recommendation for web-based cooperative learning: Learning effect and partner preference. In annиal meeting of National Association for Research on Science and Teaching, 2000.

[13] N.D. Oye, Mazleena Salleh, and N. A. Iahad. E-learning methodologies and tools. International Journal of Advanced Computer Science and Applications, 2012.

[14] B. Roy. Multicriteria Methodology for Decision Aiding. Kluwer Academic, Dordrecht, 1996.

[15] Lynda Tamine-Lechani, Mohand Boughanem, and Mariam Daoud. Evaluation of contextual information retrieval effectiveness: Overview of issues and research. Knowl. Inf. Syst., 24(1):1-34, July 2010.

[16] D.-Y. Wang, S. S. Lin, and C.-T. Sun. Diana: A computer- supported heterogeneous grouping system for teachers to conduct successful small learning groups. Computers in Human Behavior, 23(4):1997-2010, 1994.

[17] Ning Wang, Marie-Hélène Abel, Jean-Paul A. Barthès, and Elsa Negre. Trace-based computer supported cooperative work as support for learners group design. In 21th IEEE Int. Conf. on Computer Supported Cooperative Work in Design, CSCWD, New Zealand, May, 2017.

${ }^{3}$ http://memorae.hds.utc.fr/ 